\title{
Ovarialkarzinom
}

\section{PARP-Inhibitoren als Behandlungsoption in der Rezidivsituation?}

\author{
Patientinnen mit Ovarialkarzinom können in der Rezidivsituation von der Therapie mit PARP-Inhibitoren profitieren. Darauf weisen aktuelle \\ Phase-II-Studienergebnisse hin (Lederman JA et al., 2011, J Clin Oncol 29 [Suppl]:\#5003; Penson RT et al., 2011, J Clin Oncol 29 [Suppl]: \#5004; \\ Birrer M et al., 2011, J Clin Oncol 29 [Suppl]: \#5005).
}

\begin{abstract}
Die Poly-[Adenosin-Diphosphat-Ribose]-Polymerase (PARP) ist ein Schlüsselenzym für die Reparatur von DNS-Schäden. PARP-Inhibitoren bewirken allgemein, dass DNS-Einzelstrangbrüche nicht mehr repariert werden und DNS-Doppelstrangbrüche zunehmen. Bei beeinträchtigter homologer Rekombination, mittels der die DNS-Schäden ebenfalls repariert werden könnten, kommt es zur Chromosomeninstabilität und zum Zelltod - der sogenannten synthetischen Letalität. Der Einsatz von PARP-Inhibitoren zur Ovarialkarzinom-Behandlung ist vielversprechend, weil z.B. bei sporadischen serösen Ovarialkarzinomen mehr als die Hälfte der molekularen Veränderungen mit einem Defekt der homologen Rekombination assoziiert sind.
\end{abstract}

\section{Erhaltungstherapie verlängert progressionsfreies Überleben} Der PARP-Inhibitor Olaparib wurde in einer randomisierten, placebokontrollierten, doppelblinden Phase-II-Studie als Erhaltungstherapie untersucht. Die Patientinnen in dieser Studie hatten ein platinsensitives, hochgradiges, seröses Ovarialkarzinom und waren zuvor mit mindestens zwei platinhaltigen Therapien behandelt worden. Die letzte platinbasierte Chemotherapie musste dabei zu einem anhaltenden Therapieansprechen geführt haben. In dieser Situation werden die Patientinnen laut Prof. Dr. Jonathan A. Lederman, London/ Großbritannien, standardmäßig beobachtet, bis die Krankheit erneut fortschreitet. Es bedürfe jedoch dringend neuer Therapien, um die erneute Krankheitsprogression zu verzögern.

Die Erhaltungstherapie mit dem PARP-Inhibitor reduzierte das Risiko für eine Krankheitsprogression verglichen mit Placebo signifikant (Abb.). Die Risikoreduktion bestand in allen vorab definierten Subgruppen und war demzufolge z.B. unabhängig von Alter, BRCAMutationsstatus, Qualität des Ansprechens auf die vorangegangene Chemotherapie (partielle bzw. komplette Remission) und Zeit bis zur Krankheitsprogression nach der vorletzten platinbasierten Chemotherapie. Reife Daten für das Gesamtüberleben liegen noch nicht vor.

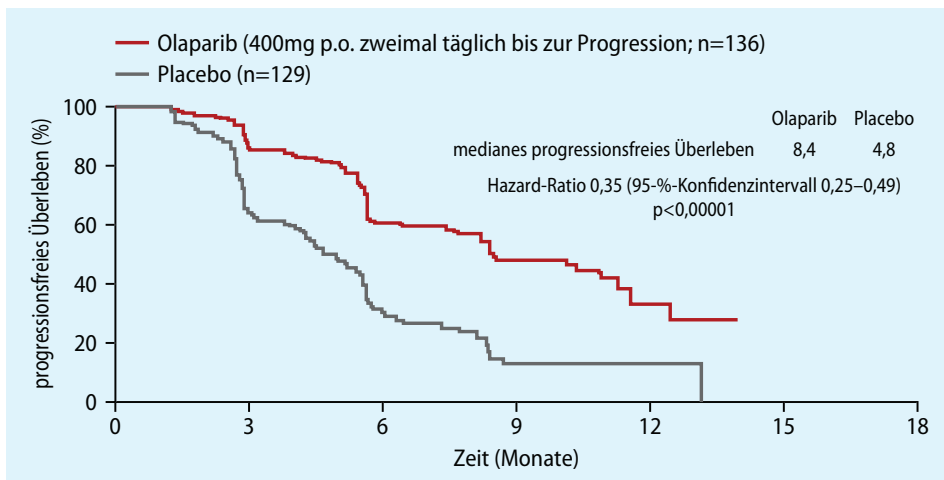

nach Lederman JA et al, 2011, J Clin Oncol 29 (Suppl):\#5003

Erhaltungstherapie mit Olaparib: Progressionsfreies Überleben
Die Behandlung mit Olaparib wurde insgesamt gut vertragen; häufige Nebenwirkungen waren Übelkeit, Erbrechen, Fatigue und Anämie.

\section{Kombinationstherapie ist aktiv}

Die Kombinationstherapie mit Iniparib plus Gemcitabin/Carboplatin wurde in zwei einarmigen Phase-II-Studien mit ähnlichem Design bei platinsensitivem bzw. platinresistentem Ovarialkarzinomrezidiv untersucht. Eingeschlossen werden konnten Patientinnen mit messbarer Erkrankung, wobei Patientinnen mit platinsensitivem Ovarialkarzinomrezidiv in der Rezidivsituation keine Chemotherapie erhalten haben durften und Patientinnen mit platinresistentem Ovarialkarzinom mindestens eine, höchstens jedoch zwei vorangegangene Chemotherapien erhalten haben sollten. Die Kombinationstherapie wurde wie folgt dosiert: Gemcitabin $\left(1.000 \mathrm{mg} / \mathrm{m}^{2} \mathrm{KOF}\right)$ an Tag 1 und 8, Carboplatin (AUC 4) an Tag 1 und Iniparib (5,6mg/kg KG) an Tag 1, 4, 8 und 11, jeweils alle 21 Tage. Es wurde zunächst für sechs Zyklen behandelt, weitere vier Zyklen und eine Erhaltungstherapie mit Iniparib bis zur Progression waren möglich. Primärer Endpunkt war die Gesamtansprechrate.

Die Gesamtansprechrate betrug bei Patientinnen mit platinsensitivem Ovarialkarzinomrezidiv 65\%: 25 von 40 Patientinnen erreichten eine partielle Remission. Das Ansprechen auf die Therapie wurde durch den BRCA-Mutationsstatus laut Dr. Richard T. Penson, Boston/ USA, nicht beeinflusst. Im Median überlebten die Patientinnen 9,5 Monate progressionsfrei. Die Zwischenauswertung bei Patientinnen mit platinresistentem Ovarialkarzinomrezidiv ergab eine Gesamtansprechrate von 25\%: 8 von 32 bislang auswertbaren Patientinnen erreichten eine partielle Remission. Bei 16 Patienten kam es zur Krankheitsstabilisierung. Auch in dieser Studie sprachen sowohl Patientinnen mit BRCA-Mutationen als auch Patientinnen mit BRCA-Wildtyp auf die Therapie an, so Dr. Michael J. Birrer, Boston/USA. In beiden Studien wurden insbesondere hämatologische Toxizitäten beobachtet, wie Neutropenie, Thrombozytopenie und Anämie.

\section{Viele offene Fragen}

Wie Prof. Dr. Stanley B. Kaye, London/Großbritannien, in der Diskussion der Studienergebnisse schlussfolgerte, sollten PARP-Inhibitoren als Behandlungsoption bei familiärem und sporadischem Ovarialkarzinom weiter untersucht werden. PARP-Inhibitoren könnten den Effekt einer Chemotherapie verstärken, so Kaye. Wichtige Fragen, auf die es in weiteren Studien Antworten zu finden gelte, sind z.B. Fragen nach der Langzeittoxizität, dem Einfluss einer Erhaltungstherapie mit PARP-Inhibitoren auf nachfolgende Chemotherapien, Biomarkern, der optimalen Dosierung bzw. dem optimalen Schema für den Einsatz von PARP-Inhibitoren in der Kombination mit einer Chemotherapie und dem Effekt der Kombinationstherapie auf das progressionsfreie Überleben und das Gesamtüberleben. $A M$ 